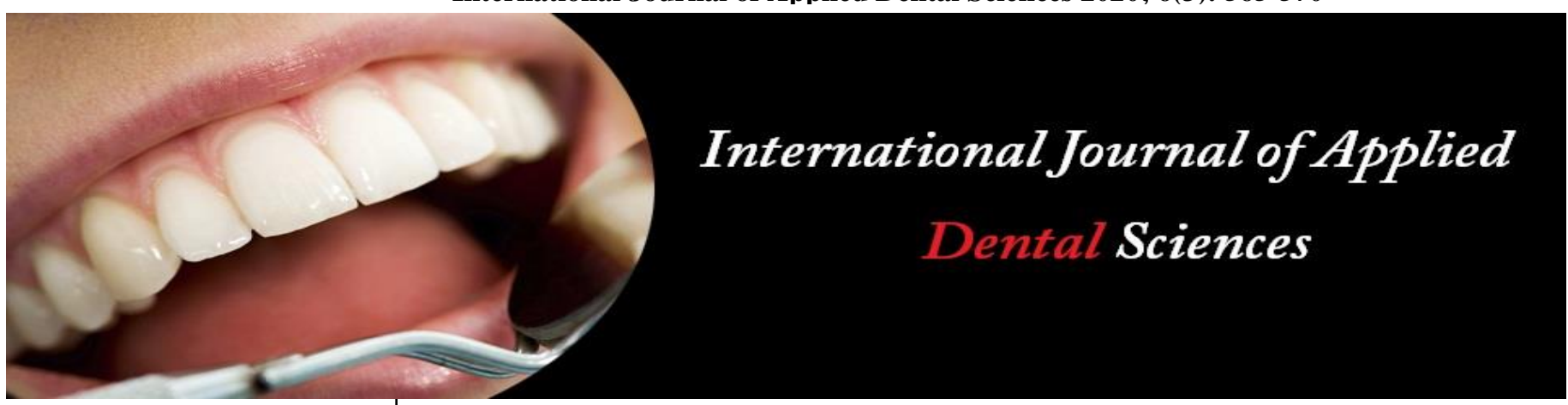

ISSN Print: 2394-7489

ISSN Online: $2394-7497$

IJADS 2020; 6(3): 565-570

(C) 2020 IJADS

www.oraljournal.com

Received: 04-06-2020

Accepted: 06-07-2020

Dr. Bosy Thankam Mathew

JSS Dental College

Bannimantap, Mysore

Karnataka, India

Dr. Girish Kuma

Lecturer, JSS Dental College

Mysore, Karnataka, India
Corresponding Author: Dr. Bosy Thankam Mathew JSS Dental College Bannimantap, Mysore, Karnataka, India

\section{Class III malocclusion with anterior open bite, orthodontic treatment in an adult patient: Case report}

\section{Dr. Bosy Thankam Mathew and Dr. Girish Kumar}

DOI: https://doi.org/10.22271/oral.2020.v6.i3i.1008

\section{Abstract}

Background: Patients with anterior open bite appear both dentoalveolar component and with increased skeletal vertical dimension.

Objectives: The main objective was to treat open bite with an ideal overbite and overjet relationships and to maintain proper intercuspstion with midline correction.

Results: The goal of the treatment has been achieved according to patients' desire and satisfactory interdigitation. In further follow up no relapse has been seen.

Conclusion: The treatment resulted in an aesthetic, functional, and stable occlusion, along with an improved facial profile.

Keywords: Open bite, orthodontic treatment, adult patient

\section{Introduction}

Open bite is an anomaly with distinct characteristics which, in addition to involving complex, multiple etiologic factors, entails aesthetic and functional consequences ${ }^{[1]}$. There are many reasons for the occurrence of open bite, including skeletal abnormal growth pattern; dental, respiratory, neurologic, and habitual factors; and tongue posture and function ${ }^{[3]}$. The prevalence varies between different populations $16 \%$ in black people and $4 \%$ in white people [7]. Types include are anterior open bite, posterior open bite, dental open bite, skeletal open bite. Anterior open bite is one of the most difficult problems to treat in orthodontics ${ }^{[3]}$. The side effects include: Aesthetics, Speech, Eating, Tooth wear. various approaches employed to treat open bite: palatal crib, orthopedic forces, extrusion of anterior teeth, MEAW technique, bite blocks to inhibit molar eruption, high-pull headgear, camouflage with or without extractions, miniimplants or mini-plates, and orthognathic surgery ${ }^{[1,3]}$. This report presents the treatment and long-term stability of an adult case of a severe anterior open bite treated by means of non - extraction treatment

\section{Diagnosis and Etiology}

A 23-year-old female with no relevant medical history, presented with the chief complaint of forwardly placed upper front teeth. On clinical examination she had a convex profile, incompetent lips with Class I molar relation on skeletal class II jaw bases with a severe anterior open bite and spacing in the upper and lower anterior region(fig:1.a). Overjet and overbite were $10 \mathrm{~mm}$ and $-4 \mathrm{~mm}$, respectively, and both canine and molar keys showed class I relation (fig: 1.b). The cephalometric analysis (fig: 1.c) showed that the patient had a divergent facial pattern with a moderate skeletal Class II relationship with ANB angle of 7 degree. Prognathic maxilla, orthognathic mandible with proclined upper and lower anteriors (table1). OPG shows that all permanent teeth erupted.

\section{Treatment Objectives}

- To achieve a proper lip competency,

- Proper anterior overbite and overjet relationship and

- Class I canine and molar,

- Aesthetically pleasing profile and functionally stable occlusion. 


\section{Treatment Plan}

- Extraction plan: Non extraction treatment plan

- Appliances: PEA Mechanotherapy with MBT 0.022 slot prescription
- Special anchorage: Transpalatal arch

- Proposed retention: Essix clear retainers in upper and lower arch
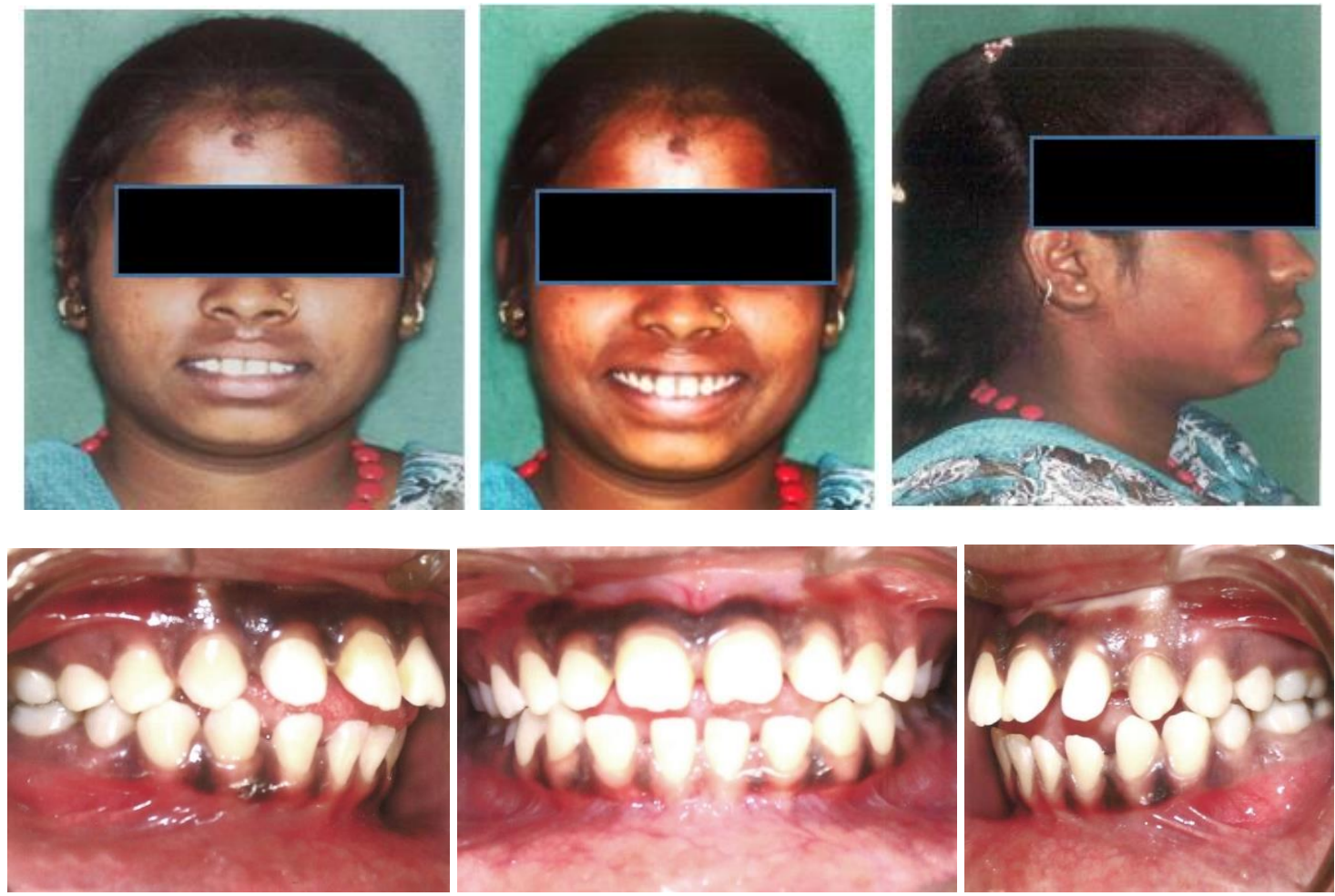

Fig 1a: Pre-treatment extraoral and intraoral photographs
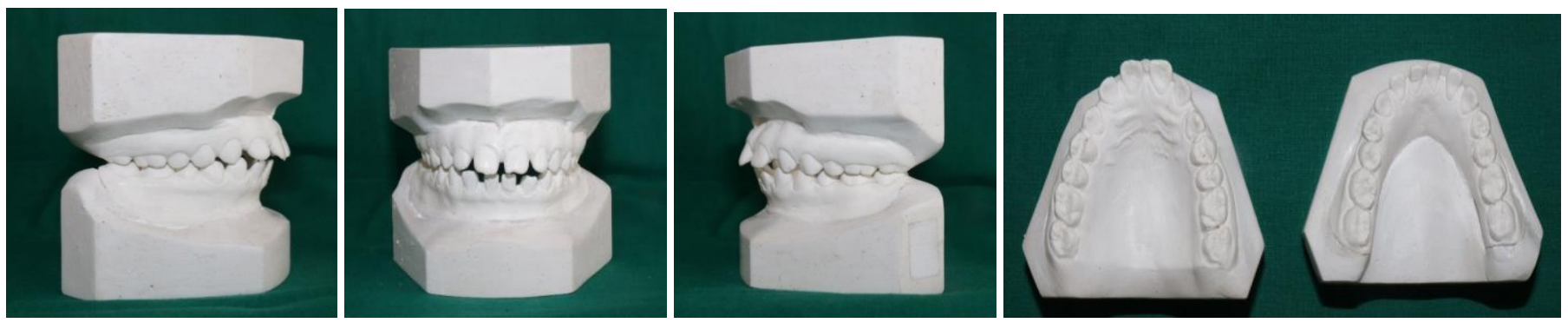

Fig 1.b: Pre-treatment patient model

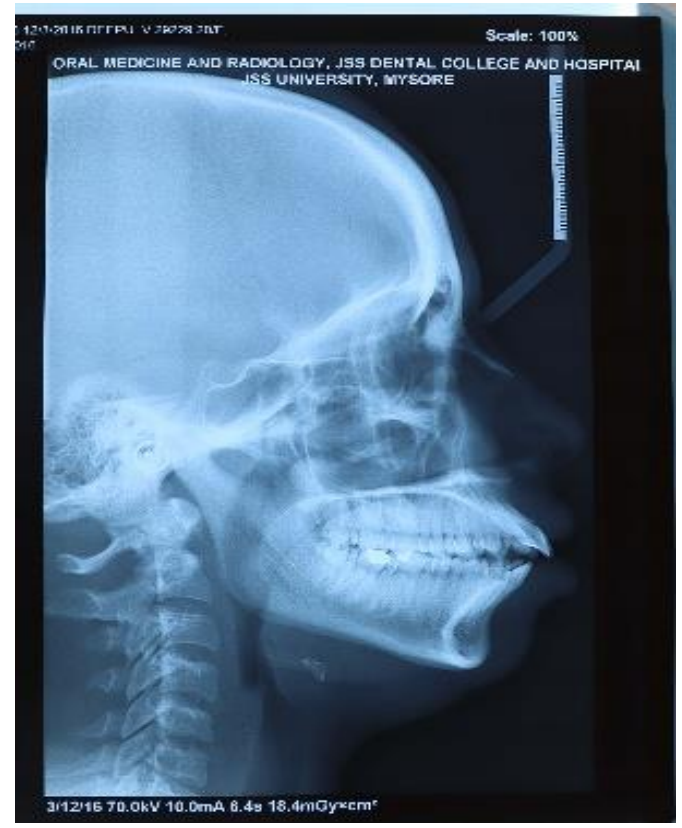

Fig 1c: Pre-treatement radiograph 


\section{Cephalometric Analysis}

Table 1: Pre-treatment cephalometric analysis

\begin{tabular}{|c|c|c|}
\hline Variable & Pretreatment & Normal \\
\hline SNA & $86^{\circ}$ & $82^{\circ} \pm 3$ \\
\hline SNB & $79^{\circ}$ & $80^{\circ} \pm 3$ \\
\hline ANB & $7^{\circ}$ & $3^{\circ} \pm 1$ \\
\hline Wits appraisal & AO ahead of BO by $10 \mathrm{~mm}$. & $0 \mathrm{~mm}$ \\
\hline $\mathrm{N} \perp \mathrm{Pt} A$ & $9 \mathrm{~mm}$ & $0 \pm 2 \mathrm{~mm}$ \\
\hline $\mathrm{N} \perp \operatorname{Pog}$ & $2 \mathrm{MM} \mathrm{mm}$ & 0 to $-4 \mathrm{~mm}$ \\
\hline Angle of inclination & 80 & $85^{\circ}$ \\
\hline Go-Gn to SN & $32^{\circ}$ & $32^{\circ}$ \\
\hline Eff. Max. Length & $87 \mathrm{~mm}$ & $93.6 \pm 3.2$ \\
\hline Eff. Mand. Length & 104 & $121.6 \pm 4.5$ \\
\hline Y-Axis & $70^{\circ}$ & $66^{\circ}$ \\
\hline Facial axis & $0^{\circ}$ & $0^{\circ}$ \\
\hline Upper incisor - NA & $8 \mathrm{~mm}$ & $4 \mathrm{~mm}$ \\
\hline Upper incisor - NA & $30^{\circ}$ & $22^{\circ}$ \\
\hline Upper incisor $-\mathrm{SN}$ & $118^{\circ}$ & $102^{\circ} \pm 2$ \\
\hline Upper incisor to maxillary plane angle & $53^{\circ}$ & $70^{\circ} \pm 5$ \\
\hline Lower incisor to mandibular plane angle & $\circ$ & $90^{\circ} \pm 3$ \\
\hline Lower incisor to NB & $3 \mathrm{~mm}$ & $4 \mathrm{~mm}$ \\
\hline Lower incisor to NB & $19^{\circ}$ & $25^{\circ}$ \\
\hline Interincisal angle & $122^{\circ}$ & $133^{\circ} \pm 10$ \\
\hline Maxillary mandibular planes angle & $17^{\circ}$ & $27^{\circ} \pm 5$ \\
\hline Lower anterior face height & $59 \mathrm{~mm}$ & $67.2 \pm 4.7 \mathrm{~mm}$ \\
\hline Face height ratio & $69 \%$ & $62-65 \%$ \\
\hline Lower incisor to APO line & $3 \mathrm{~mm}$ & $1-2 \mathrm{~mm}$ \\
\hline
\end{tabular}

\section{Treatment Progress}

PEA appliance included $0.022 \times 0.028$ inch MBT prescription. The arch wire sequence used were as follows: 0.016 inch $\mathrm{NiTi}$, followed by $0.016 \mathrm{SS}, 0.017 \times 0.025$ inch $\mathrm{NiTi}$, box elastics were given engaging from upper left to right canine to lower left to lower right canine, followed by $0.017 \times 0.025 \mathrm{SS}$, $0.019 x 0.025 \mathrm{SS}$ wires were placed and post retraction settling elastics placed (fig 2).
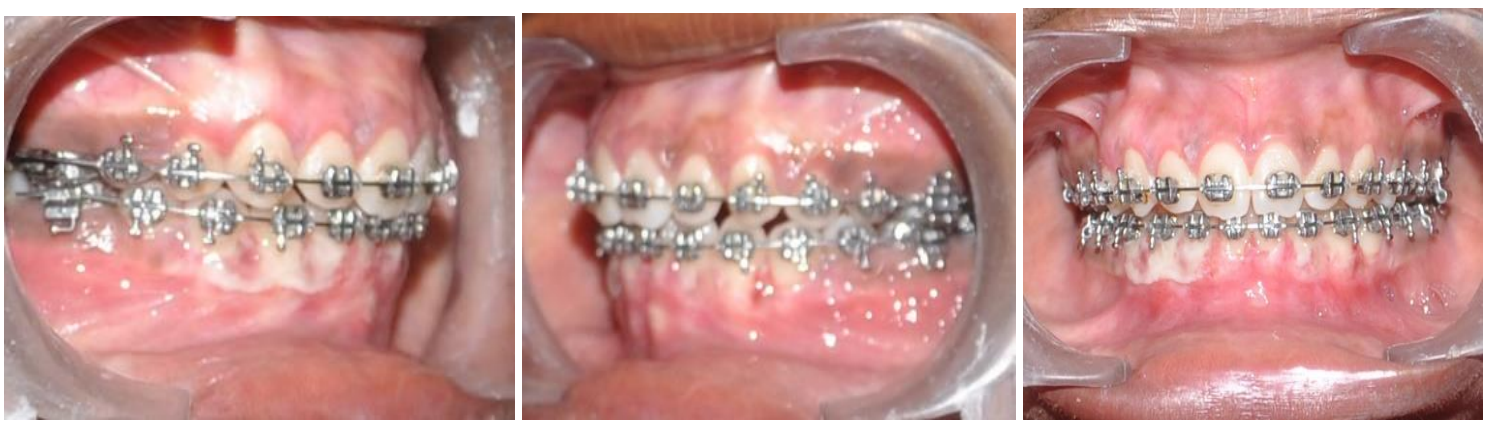

Fig 2: Mid treatment intraoral photographs

\section{Treatment Results}

An ideal overjet and overbite of $2 \mathrm{~mm}$ was achieved with adequate intercuspation, with angles Class I molar relationship on right and left side, Class I canine relationship, Class I incisor relationship, normal lateral and protrusive excursions was achieved (fig 3.b). Facial appearance and profile improved as a result of dental and skeletal changes (fig 3.a). Orthodontic treatment was done to correct crowding of upper and lower anteriors. PEA mechanotherapy was used with sequential stripping, to correct crowding. A satisfactory result and a good interception have been achieved.
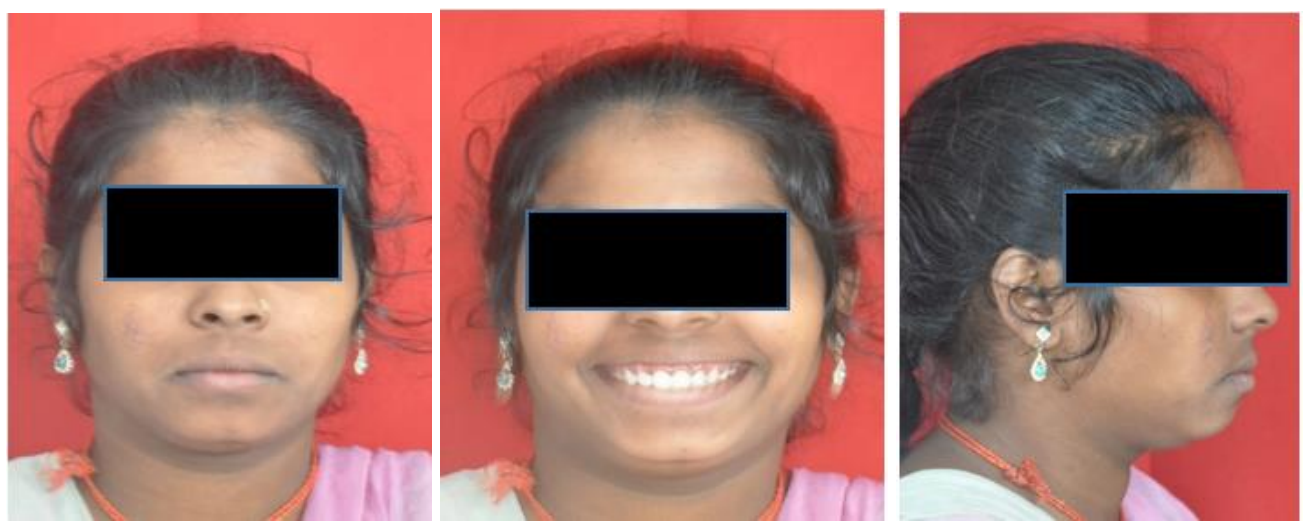

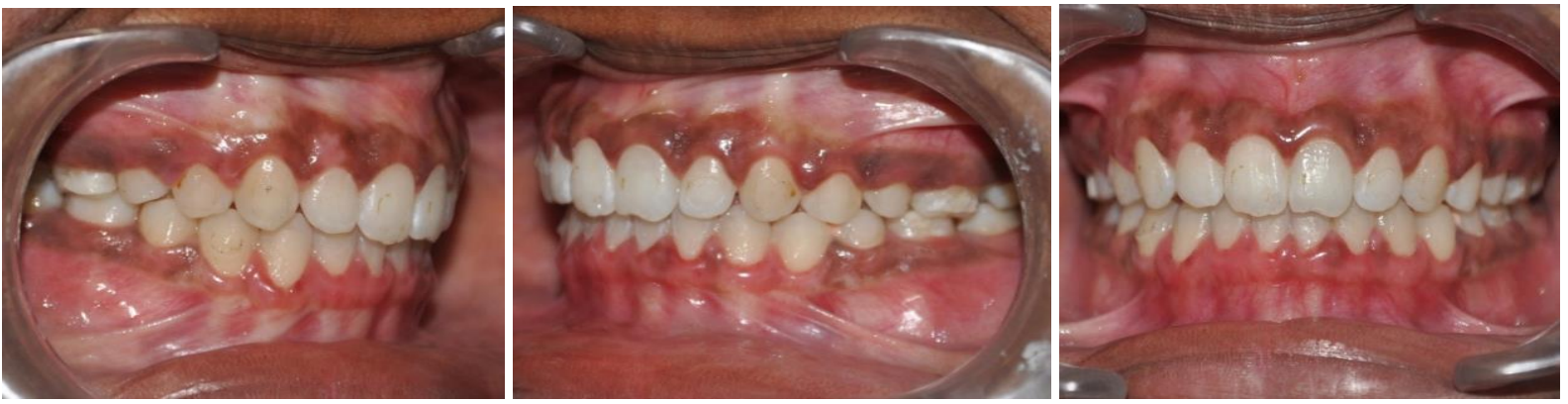

Fig 3a: Post-treatment extraoral and intraoral photographs
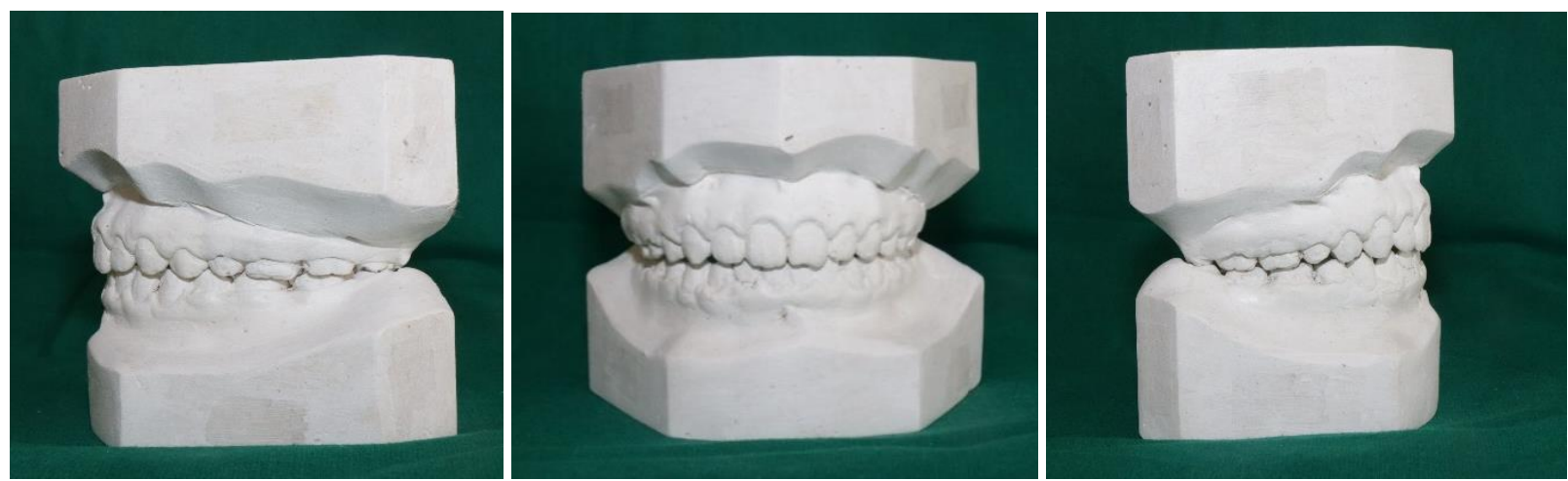

Fig 3: 3b Post-treatment patient model

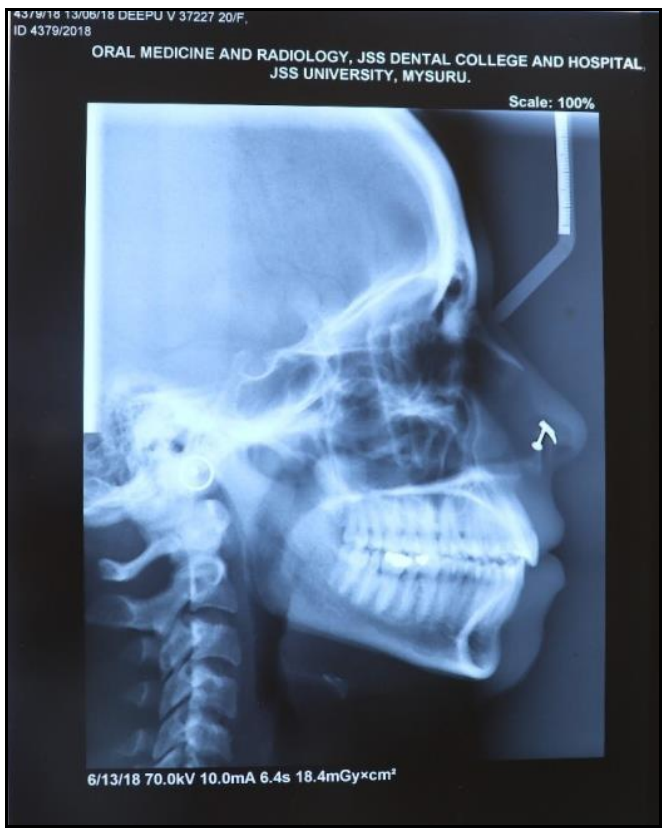

Fig 3: 3.c Post-treatment Radiograph

\section{Cephalometric Analysis}

Table 2: post-treatment cephalometric analysis

\begin{tabular}{|c|c|c|}
\hline Variable & Posttreatment & Normal \\
\hline SNA & $86^{\circ}$ & $82^{\circ} \pm 3$ \\
\hline SNB & $80^{\circ}$ & $80^{\circ} \pm 3$ \\
\hline ANB & $6^{\circ}$ & $3^{\circ} \pm 1$ \\
\hline Wits appraisal & AO AHEAD OF BO by $2 \mathrm{~mm}$. & $0 \mathrm{~mm}$ \\
\hline $\mathrm{N} \perp$ Pt A & $-4 \mathrm{~mm}$ & $0 \pm 2 \mathrm{~mm}$ \\
\hline $\mathrm{N} \perp$ Pog & $84^{\circ}$ & 0 to $-4 \mathrm{~mm}$ \\
\hline Angle of inclination & $30^{\circ}$ & $85^{\circ}$ \\
\hline Go-Gn to SN & $87 \mathrm{~mm}$ & $32^{\circ}$ \\
\hline Eff. Max. Length & $104 \mathrm{~mm}$ & $93.6 \pm 3.2$ \\
\hline Eff. Mand. Length & $68^{\circ}$ & $121.6 \pm 4.5$ \\
\hline Y- Axis & $-2^{\circ}$ & $66^{\circ}$ \\
\hline Facial axis & $4 \mathrm{~mm}$ & $0^{\circ}$ \\
\hline Upper incisor - NA & & $4 \mathrm{~mm}$ \\
\hline
\end{tabular}

$\sim 568 \sim$ 


\begin{tabular}{|c|c|c|}
\hline Upper incisor - NA & $16^{\circ}$ & $22^{\circ}$ \\
\hline Upper incisor - SN & $100^{\circ}$ & $102^{\circ} \pm 2$ \\
\hline Upper incisor to maxillary plane angle & $74^{\circ}$ & $70^{\circ} \pm 5$ \\
\hline Lower incisor to mandibular plane angle & $92^{\circ}$ & $90^{\circ} \pm 3$ \\
\hline Lower incisor to NB & $4 \mathrm{~mm}$ & $4 \mathrm{~mm}$ \\
\hline Lower incisor to NB & $29^{\circ}$ & $25^{\circ}$ \\
\hline Interincisal angle & $129^{\circ}$ & $133^{\circ} \pm 10$ \\
\hline Maxillary mandibular planes angle & $27^{\circ}$ & $27^{\circ} \pm 5$ \\
\hline Lower anterior face height & $59 \mathrm{~mm}$ & $67.2 \pm 4.7 \mathrm{~mm}$ \\
\hline Face height ratio & $66.6 \%$ & $62-65 \%$ \\
\hline Lower incisor to APO line & $-3 \mathrm{~mm}$ & $1-2 \mathrm{~mm}$ \\
\hline & & \\
\hline
\end{tabular}

Table 3: 3 c comparison of pre and post treatment cephalometric analysis

\begin{tabular}{|c|c|c|c|}
\hline Variable & Pretreatment & Posttreatment & Difference \\
\hline SNA & $86^{\circ}$ & $86^{\circ}$ & $0^{\circ}$ \\
\hline SNB & $79^{\circ}$ & $80^{\circ}$ & $1^{\circ}$ \\
\hline ANB & $7^{\circ}$ & $6^{\circ}$ & $8 \mathrm{~mm}$ \\
\hline Wits appraisal & AO ahead of BO by 10mm. & AO AHEAD OF BO by 2mm. & $4 \mathrm{~mm}$ \\
\hline N P Pt A & $9 \mathrm{~mm}$ & $-4 \mathrm{~mm}$ & $-2 \mathrm{~mm}$ \\
\hline N P.g & $2 \mathrm{~mm}$ & $84^{\circ}$ & $4^{\circ}$ \\
\hline Angle of inclination & 80 & $30^{\circ}$ & $2^{\circ}$ \\
\hline Go-Gn to SN & $32^{\circ}$ & $87 \mathrm{~mm}$ & $0 \mathrm{~mm}$ \\
\hline Eff. Max. Length & $87 \mathrm{~mm}$ & $104 \mathrm{~mm}$ & $2^{\circ}$ \\
\hline Eff. Mand. Length & 104 & $68^{\circ}$ & $-2^{\circ}$ \\
\hline Y- Axis & $70^{\circ}$ & $-2^{\circ}$ & $4 \mathrm{~mm}$ \\
\hline Facial axis & $0^{\circ}$ & $4 \mathrm{~mm}$ & $14^{\circ}$ \\
\hline Upper incisor - NA & $8 \mathrm{~mm}$ & $16^{\circ}$ & $18^{\circ}$ \\
\hline Upper incisor - NA & $30^{\circ}$ & $100^{\circ}$ & $21^{\circ}$ \\
\hline Upper incisor - SN & $118^{\circ}$ & $74^{\circ}$ & $21^{\circ}$ \\
\hline Upper incisor to maxillary plane angle & $53^{\circ}$ & $92^{\circ}$ & $1 \mathrm{~mm}$ \\
\hline Lower incisor to mandibular plane angle & $113^{\circ}$ & $4 \mathrm{~mm}$ & $10^{\circ}$ \\
\hline Lower incisor to NB & $3 \mathrm{~mm}$ & $29^{\circ}$ & $7^{\circ}$ \\
\hline Lower incisor to NB & $19^{\circ}$ & $129^{\circ}$ & $10^{\circ}$ \\
\hline Interincisal angle & $122^{\circ}$ & $27^{\circ}$ & $0 \mathrm{~mm}$ \\
\hline Maxillary mandibular planes angle & $17^{\circ}$ & $59 \mathrm{~mm}$ & $2.4 \%$ \\
\hline Lower anterior face height & $59 \mathrm{~mm}$ & $66.6 \%$ & $0 \mathrm{~mm}$ \\
\hline Face height ratio & $69 \%$ & $-3 \mathrm{~mm}$ & \\
\hline Lower incisor to APO line & $3 \mathrm{~mm}$ & & \\
\hline
\end{tabular}

\section{Discussion}

Combination of fixed orthodontic treatment and vertical class II elastics is implemented to obtain a stable result for treatment. During diagnosis, the vertical dimension with skeletal morphology need to measure properly for determining that the open bite whether it is dental or skeletal [8 9]. Proper diagnosis, treatment planning, and retention are critical to achieve the most stable and favourable out-comes for patients with open bite malocclusion ${ }^{[10]}$. This case report showed the patient had an anterior open bite with dental component. Treatment for this patient includes maintenance of ideal overbite and overjet relationships, proper intercuspation with midline correction. After finishing the treatment removable orthodontic retainer was provided with proper instructions.

\section{Conclusion}

Open bite is an anomaly with distinct, easily recognizable features that can be found in $25 \%$ to $38 \%$ of orthodontic patients The present case report details the non-surgical orthodontic treatment of an adult patient with a complex anterior open-bite treated using a combination of fixed appliances, and vertical intermaxillary elastics.

\section{References}

1. Mírian Aiko Nakane Matsumoto. Angle Class I malocclusion, with anterior open bite, treated with extraction of permanent teeth. Dental Press J Orthod 2011; 16(1):126-38.

2. Nitin VM, Abhishek Ranjan, Raghunath NM. Treatment of Skeletal Class III Malocclusion with Anterior Open Bite using Ortho-Surgical approach: A Case Report. Int J Oral Health Med Res. 2017; 4(3):60-64.

3. Alev Yilmaz, Ayc, a Arman-O” zc, irpic. Camouflage Treatment of a Severe Open Bite Case. Turkish J Orthod. 2014; 27:9-15.

4. Isaacson JR, Isaacson RJ, Speidel TM, Worms FW. Extreme variation in vertical facial growth and associated variation in skeletal and dental relations. Angle Orthod. 1971; 41:219-229.

5. Schendel SA, Eisenfeld J, Bell WH, Epker BN, Mishelevich DJ. The long-face syndrome: vertical maxillary excess. Am J Orthod. 1976; 70:398-408

6. Katsaros C, Berg R. Anterior open bite malocclusion: A follow-up study of orthodontic treatment effects. Eur J Orthod. 1993; 15:273-280

7. Proffit WR, White RP. "Who needs surgical-orthodontic treatment?". The International Journal of Adult Orthodontics and Orthognathic Surgery. 1990; 5(2):81-89

8. Ngan P, Fields HW. Open bite: a review of etiology and management. Pediatr Dent 1997; 19:91-8.

9. Alam MK, Basri R, Kathiravan P et al. Cephalometric evaluation for Bangladeshi adult by Steiner analysis. Int Med J. 2012; 19:262-265. 
10. Atsawasuwan P, Hohlt W, Evans CA. Nonsurgical approach to class I open-bite mal-occlusion with extrusion mechanics: a 3-year retention case report. Am J Orthod Dentofacial Orthop. 2015; 147:499-508 